\title{
The clinical relevance of the IBD-associated variation within the risk gene locus encoding protein tyrosine phosphatase non-receptor type 2 in patients of the Swiss IBD cohort
}

Spalinger, Marianne R ; Voegelin, Marius ; Biedermann, Luc ; Zeitz, Jonas ; Rossel, Jean-Benoit ; Sulz, Michael Christian ; Frei, Pascal ; Scharl, Sylvie ; Vavricka, Stephan R ; Fried, Michael ; Rogler, Gerhard ; Scharl, Michael ; Swiss IBD Cohort Study Group

\begin{abstract}
BACKGROUND/AIMS The single nucleotide polymorphism (SNP) rs1893217 within the gene locus encoding protein tyrosine phosphatase non-receptor type 2 (PTPN2) results in a dysfunctional PTPN2 protein is associated with Crohn's disease (CD) and exists in perfect linkage disequilibrium with the CD- and ulcerative colitis (UC)-associated PTPN2 SNP rs2542151. We investigated associations of PTPN2 SNP rs1893217 and clinical characteristics of inflammatory bowel disease (IBD) patients. METHODS One thousand seventy three patients with CD and 734 patients with UC from the Swiss IBD Cohort Study (SIBDCS) were included. Epidemiologic, disease and treatment characteristics were analysed for an association with the presence of one of the rs1893217 genotypes 'homozygous wild-type' (TT), 'heterozygous' (CT) and 'homozygous variant' (CC). RESULTS About 2.88\% of IBD patients were identified with CC, $26.8 \%$ with CT and $70.4 \%$ with TT genotype. The CC-genotype was associated with the existence of gallstones in CD and pancolitis in UC patients. The presence of the C-allele (i.e. either $\mathrm{CC}$ or CT genotype) was associated with the onset of uveitis, but protected from aphthous oral ulcers in $\mathrm{CD}$ patients. UC patients carrying a $\mathrm{C}$-allele were diagnosed at an older age but required intestinal surgery more often. The presence of the $\mathrm{C}$-allele was associated with a successful treatment with antiTNF antibodies in both CD and UC patients. CONCLUSION IBD patients carrying the C-allele of PTPN2 SNP rs1893217 are at greater risk for developing a severe disease course but are more likely to respond to treatment with anti-TNF antibodies. These findings demonstrate a clinical relevance of this PTPN2 risk variant in IBD patients.
\end{abstract}

DOI: https://doi.org/10.1159/000444479

Posted at the Zurich Open Repository and Archive, University of Zurich

ZORA URL: https://doi.org/10.5167/uzh-134501

Journal Article

Published Version

Originally published at:

Spalinger, Marianne R; Voegelin, Marius; Biedermann, Luc; Zeitz, Jonas; Rossel, Jean-Benoit; Sulz, Michael Christian; Frei, Pascal; Scharl, Sylvie; Vavricka, Stephan R; Fried, Michael; Rogler, Gerhard; Scharl, Michael; Swiss IBD Cohort Study Group (2016). The clinical relevance of the IBD-associated variation within the risk gene locus encoding protein tyrosine phosphatase non-receptor type 2 in patients of the Swiss IBD cohort. Digestion, 93(3):182-192.

DOI: https://doi.org/10.1159/000444479 


\title{
The Clinical Relevance of the IBD-Associated Variation within the Risk Gene Locus Encoding Protein Tyrosine Phosphatase Non-Receptor Type 2 in Patients of the Swiss IBD Cohort
}

\author{
Marianne R. Spalinger ${ }^{a}$ Marius Voegelin ${ }^{a}$ Luc Biedermann $^{a}$ \\ Jonas Zeitz ${ }^{a}$ Jean-Benoit Rossel ${ }^{c}$ Michael Christian Sulz ${ }^{a}{ }^{d}$ Pascal Frei $^{a}$ \\ Sylvie Scharla Stephan R. Vavricka ${ }^{a, b}$ Michael Fried ${ }^{a, b} \quad$ Gerhard Rogler $^{a, b}$ \\ Michael Scharla, b for the Swiss IBD Cohort Study Group \\ ${ }^{a}$ Division of Gastroenterology and Hepatology, University Hospital Zurich, and ${ }^{\text {b } Z u r i c h ~ C e n t e r ~ f o r ~ I n t e g r a t i v e ~}$ \\ Human Physiology, University of Zurich, Zurich, 'Institute of Social and Preventive Medicine, Université de \\ Lausanne, Lausanne, and d Division of Gastroenterology and Hepatology, Kantonsspital St. Gallen, St. Gallen, \\ Switzerland
}

\section{Key Words}

Inflammatory bowel disease $\cdot$ Protein tyrosine phosphatase non-receptor type $2 \cdot$ Genetic polymorphisms $\cdot$ Anti-TNF antibodies · Pancolitis

\footnotetext{
Abstract

Background/Aims: The single nucleotide polymorphism (SNP) rs1893217 within the gene locus encoding protein tyrosine phosphatase non-receptor type 2 (PTPN2) results in a dysfunctional PTPN2 protein is associated with Crohn's disease $(C D)$ and exists in perfect linkage disequilibrium with the CD- and ulcerative colitis (UC)-associated PTPN2 SNP rs2542151. We investigated associations of PTPN2 SNP rs 1893217 and clinical characteristics of inflammatory bowel disease (IBD) patients. Methods: One thousand seventy three patients with $C D$ and 734 patients with UC from the Swiss IBD Cohort Study (SIBDCS) were included. Epidemiologic, disease and treatment characteristics were analysed for an association with the presence of one of the rs 1893217
}

genotypes 'homozygous wild-type' (TT), 'heterozygous' (CT) and 'homozygous variant' (CC). Results: About $2.88 \%$ of IBD patients were identified with CC, $26.8 \%$ with $\mathrm{CT}$ and $70.4 \%$ with $T$ T genotype. The CC-genotype was associated with the existence of gallstones in $C D$ and pancolitis in UC patients. The presence of the C-allele (i.e. either CC or CT genotype) was associated with the onset of uveitis, but protected from aphthous oral ulcers in CD patients. UC patients carrying a $\mathrm{C}$-allele were diagnosed at an older age but required intestinal surgery more often. The presence of the $\mathrm{C}$-allele was associated with a successful treatment with anti-TNF antibodies in both CD and UC patients. Conclusion: IBD patients carrying the C-allele of PTPN2 SNP rs1893217 are at greater risk for developing a severe disease course but are more likely to respond to treatment with anti-TNF antibodies. These findings demonstrate a clinical relevance of this PTPN2 risk variant in IBD patients.

(C) 2016 S. Karger AG, Basel

\section{KARGER}

E-Mail karger@karger.com www.karger.com/dig
(C) 2016 S. Karger AG, Basel

$0012-2823 / 16 / 0933-0182 \$ 39.50 / 0$ 


\section{Introduction}

Recent genome-wide association studies identified $>190$ risk gene loci being associated with inflammatory bowel disease (IBD) to date $[1,2]$. One of those risk loci lies within the gene locus encoding for protein tyrosine phosphatase non-receptor type 2 (PTPN2). Originally, the PTPN2 single nucleotide polymorphism (SNP) rs2542151 had been associated with both, Crohn's disease (CD) and ulcerative colitis (UC) [3-5]. This polymorphism is in perfect linkage disequilibrium with a further SNP within the PTPN2 gene locus, namely 1893217 [6]. Using blood samples from the patient collective of the Swiss IBD Cohort Study (SIBDCS), we have previously validated this correlation in $\mathrm{CD}$ patients and demonstrated that the PTPN2 SNP rs1893217 is associated with the occurrence of CD [4]. This is of particular importance, since we have shown that the presence of the SNP rs1893217 causes dysfunction of the PTPN2 protein product [4].

On a functional level, PTPN2 plays a critical role for immune and epithelial cell function, and loss of PTPN2 activity results in a breakdown of the intestinal epithelial barrier, aberrant cytokine secretion and dysfunctional autophagy in vitro [4, 7-10]. PTPN2 knock-out mice feature severe alterations in erythropoiesis, thrombopoiesis as well as B-and T-cell function and finally die within a few weeks after birth due to progressive systemic inflammation $[11,12]$. Using mice featuring a knock-out of PTPN2 in T-cells, it has been demonstrated that PTPN2 is a critical negative regulator of $\mathrm{T}$-cell receptor signaling, and T-cell specific loss of PTPN2 promotes autoimmune and inflammatory conditions $[13,14]$.

We have recently shown that the loss of PTPN2 in CD4-positive cells results in severe colitis and the onset of systemic inflammation, for example, in the liver, kidneys and the skin, in the dextran sodium sulfate colitis as well as in the transfer colitis mouse model. Further, these mice exhibit elevated levels of dsDNA antibodies, antinuclear antibodies and anti-mitochondrial antibodies underlining the systemic inflammatory effect of PTPN2 dysfunction. Of particular interest, we found that PTPN2 dysfunction results in a dysregulation of CD4-positive Tcell subsets by enhancing levels of Th1 and Th17, but reducing levels of regulatory T-cells. These observations could be fully confirmed using serum samples from SIBDCS patients carrying the CD-associated PTPN2 risk allele. Additionally, mice featuring a loss of PTPN2 in CD4-positive T-cells featured similar alterations in the composition of the intestinal microbiota than those that can be observed in CD patients [15].

Clinical Relevance of PTPN2

Polymorphisms
These observations strongly suggest investigating whether the presence of the $\mathrm{CD}$-associated gene variant rs 1893217 is associated with certain clinical characteristics in IBD patients. To date, data about the clinical relevance of the presence of the IBD-associated risk variants are rare and a better understanding about these associations might critically contribute to a better understanding of the complex pathophysiology of IBD as well as to a better characterization and ultimately prediction of the clinical course of IBD patients, which thus might finally have an impact on treatment decisions and algorithms.

Using the unique patient collective of the SIBDCS, we aimed at investigating, whether the presence of the PTPN2 risk variant, rs1893217 is associated with distinctive disease characteristics in IBD patients from the SIBDCS.

\section{Material and Methods}

\section{Study Design}

Patient data were entirely obtained from the register of the nationwide SIBDCS, in which patients with IBD from all regions of Switzerland have prospectively been included since 2006 [16]. The cohort study is supported by the Swiss National Science Foundation and approved by the local ethical committees (IRB approval number: EK-1316, approved on February 5, 2007 by the Cantonal Ethics Committee of the Canton Zürich, Switzerland). The cohort goals and methodology are described elsewhere [16].

We included 1,843 IBD patients who were enrolled in the study at the time of data acquisition and had been previously genotyped for the CD-associated risk variant rs 1893217 within the PTPN2 gene locus. Genotyping had been performed as a large approach by which the patient collective of the SIBDC was genotyped for the presence of genetic variations in all of the currently known IBD risk loci. One thousand seventy three patients had CD, 734 had UC and 46 had indeterminate colitis (IC). Due to low patient numbers, IC patients have been excluded from the analysis, unless otherwise stated. Of note, the rs 1893217 polymorphism is in perfect linkage disequilibrium with PTPN2 polymorphism rs2542151, which has been previously associated with both, CD and UC [3, 4]. The PTPN2 polymorphism rs1893217 occurs in 3 possible genotypes: homozygous wildtype (TT), heterozygous (CT), and homozygous variant (CC). The goal of this study was to analyse whether the presence of the CT- or CC-form is associated with clinical characteristics of IBD patients.

Clinical phenotypes were classified with regard to disease location, which was stratified into 1 of 4 groups according to the Montreal classification and analysed separately for initial location and current location: ileal disease with or without disease limitation to the cecum (L1), a disease limited to the colon (L2), an ileal disease with disease of the colon beyond the cecum (L3), or disease of the upper gastrointestinal tract (L4). Patients with fistulae were classified into 4 groups: perianal fistula, other type fistula (non-perianal fistula), multiple fistulae and any type fistula. The presence of any intestinal stenosis was included in the analysis as positive for stenosis. UC was classified according to the Montreal classification [17] into proctitis (L1), left-sided colitis (L2), pancolitis (L3) or 'location 
unknown'. We also included the history of intestinal surgery. Gender, age at diagnosis, smoking history, and the presence of extraintestinal manifestations (EIM) were taken into account. We further obtained data about current and prior treatment with 5-aminosalicylate, antibiotics, steroids, immunosuppressants (namely azathioprine/6-mercaptopurine), calcineurin inhibitors (tacrolimus, cyclosporine), and anti-TNF drugs (infliximab, adalimumab, and certolizumab) at enrollment or according to the term 'ever treated with'. Anti-TNF non-response was defined as one of the following: (1) breakthrough/loss of response, (2) primary non response (never effective), (3) therapy stop due to side effects/intolerance.

The ethnic background of 1,328 of the 1,843 studied patients is known and is as follows: European: 1,234 (80.65\% of 1,530), African: 19 (1.24\%), Asian: 18 (1.18\%), Hispanic: 27 (1.76\%), Jewish: $22(1.44 \%)$, other: $8(0.52 \%)$. The remaining patients did not give us information about their ethnic background.

\section{Statistical Analysis}

Clinical data were retrieved from the data center of the SIBDCS at the University of Lausanne. These data and additional data obtained from a review of the patients' files were entered into a database (access 2000; Microsoft Switzerland Ltd., Liab., Co., Wallisellen, Switzerland). The Statistical Package for the Social Sciences (version 21; SPSS, Chicago, Ill., USA) was used for the statistical analysis.

Crude differences about the association of the PTPN2 variant in relation to fistulae, stenosis, smoking status, disease location, age at diagnosis, medications and history of intestinal resection surgery were assessed using the Pearson's chi-square test or the Fisher's exact test (Fisher's exact test used if strata comprised a sample size $\leq 5$ ). To calculate multiple logistic regression models, univariate regressions of each factor was performed and all parameters fitted together in a single model. Subsequently, variables were removed or added back one after the other in order to improve the model. In the present document, only the final models including factors contributing to significant differences are shown. A p value $<0.05$ was considered significant, and findings were corrected for multiple testing using Bonferroni correction.

\section{Ethical Considerations}

The SIBDCS is approved by the local ethical committees (IRB approval number: EK-1316, approved on February 5, 2007 by the Cantonal Ethics Committee of the Canton Zürich, Switzerland). Written informed consent was obtained before inclusion in the cohort.

\section{Results}

\section{Demographic Aspects and Disease Characteristics}

In this study, we analysed a total of 1,843 IBD patients, consisting of 1,073 (58.2\%) CD, 734 (39.8\%) UC and 36 $(2.0 \%)$ IC patients. Five hundred and thirty patients (49.4\%) being males were counted in the CD and 429 (55.7\%) in the UC/IC subgroup. Of the entire set of patients, 53 persons $(2.88 \%)$ were identified having a homozygous variant of the PTPN2 polymorphism (CC), 493
Table 1. The distribution of the 3 genotypes among CD, UC and IC patients in our data set

\begin{tabular}{lcccc}
\hline $\begin{array}{l}\text { Patients per } \\
\text { genotype, } \%\end{array}$ & CD & UC & IC & Total \\
\hline TT & $753(70.2)$ & $518(70.6)$ & $26(72.2)$ & $1,297(70.4)$ \\
CT & $282(26.3)$ & $201(27.4)$ & $10(27.8)$ & $493(26.8)$ \\
CC & $38(3.5)$ & $15(2.0)$ & 0 & $53(2.9)$ \\
Total & 1,073 & 734 & 36 & 1,843 \\
\hline
\end{tabular}

(26.8\%) carrying the heterozygous form (CT), and 1,297 (70.4\%) the homozygous wild-type (TT). According to a chi-square test, no significant difference regarding genotype distribution was detected between CD, UC and IC patients (table 1). Further, no difference regarding the distribution of the genotypes was found between different ethnic groups (online suppl. table 1; for all online suppl. material, see www.karger.com/doi/10.1159/000444479).

The median age of first diagnosis was at 25.6 in CD patients, whereas it was at 30.9 years for UC. An ileocolonic disease location (L3 according to Montreal and Vienna Classification) was with $37.1 \%$ the most commonly affected site in CD, followed by colonic (L2; $30.7 \%)$ and ileal (L1; 28.2\%) disease location. Furthermore, $377 \mathrm{CD}$ patients $(35.1 \%)$ suffered from stenosis and $461 \mathrm{CD}$ patients $(43.0 \%)$ had a fistulising disease course. In UC, $42.0 \%$ of the patients featured widespread involvement of the whole colon (pancolitis, E3 according to Montreal classification); in $42.0 \%$, the disease reached up the splenic flexure (left-sided colitis, E2), and only in $12.9 \%$, UC was limited to the anorectal region (proctitis, E1). In 3.1\%, the exact disease location remained undetermined.

About one third (36.8\%) of CD patients but only $1.7 \%$ of the UC subgroup had undergone IBD-related surgeries in the past. EIM were present in $44.0 \%$ of the patients with $\mathrm{CD}$ and in $33.4 \%$ in the group with UC. When considering all patient groups, peripheral arthritis and arthralgia were the most common EIM. Osteopenia/osteoporosis (18.7\%), adverse effect of a treatment (10.5\%) and gallstones (5.3\%) were the most frequent complications in CD patients, whereas in UC patients, osteopenia/osteoporosis $(13.1 \%)$, adverse effect of a treatment (10.4\%) and nephrolithiasis (3.0\%) were the most frequent. More detailed numbers about the occurrence of EIM and other disease-related complications, as well as an overview about patients' demographics and disease characteristics can be found in online supplementary table 2 . 
Table 2. Univariate regression analysis of $\mathrm{CD}$ patients at a $5 \%$ level to identify factors associated with the CC genotype

\begin{tabular}{|c|c|c|}
\hline Presence of CC genotype in CD patients & OR $(95 \% \mathrm{CI})$ & $\mathrm{p}$ value \\
\hline \multicolumn{3}{|l|}{ Gender } \\
\hline Male & 1 (ref.) & - \\
\hline Female & $0.912(0.473-1.758)$ & 0.783 \\
\hline Age at diagnosis (decades) & $1.315(1.068-1.620)$ & 0.010 \\
\hline \multicolumn{3}{|l|}{ Current CD location } \\
\hline L1 & 1 (ref.) & - \\
\hline $\mathrm{L} 2$ & $2.296(0.939-5.617)$ & 0.069 \\
\hline L3 & $1.427(0.562-3.621)$ & 0.455 \\
\hline \multicolumn{3}{|l|}{ Fistula or abscess (FAF_any) } \\
\hline No & 1 (ref.) & - \\
\hline Yes & $1.267(0.657-2.443)$ & 0.480 \\
\hline \multicolumn{3}{|l|}{ Stenosis (sten_any) } \\
\hline No & 1 (ref.) & - \\
\hline Yes & $1.271(0.651-2.482)$ & 0.482 \\
\hline \multicolumn{3}{|l|}{ Intestinal surgery } \\
\hline No & 1 (ref.) & - \\
\hline Yes & $1.076(0.547-2.118)$ & 0.831 \\
\hline \multicolumn{3}{|l|}{ Other medical history* } \\
\hline Psoriasis & $1.032(0.242-4.408)$ & 0.966 \\
\hline TBC & Omitted since TBC predicts 'no CC' perfectly & - \\
\hline \multicolumn{3}{|l|}{ Complications* } \\
\hline Osteopenia/osteoporosis & $0.855(0.351-2.078)$ & 0.729 \\
\hline Deep venous thrombosis & Omitted since it predicts 'no CC' perfectly & - \\
\hline Pulmonary embolism & Omitted since it predicts 'no CC' perfectly & - \\
\hline Gallstones & $4.793(2.000-11.49)$ & 0.001 \\
\hline Nephrolithiasis & $1.608(0.372-6.963)$ & 0.525 \\
\hline Malabsorption syndrome & $1.855(0.549-6.269)$ & 0.320 \\
\hline Perforation/peritonitis & $1.433(0.332-6.179)$ & 0.629 \\
\hline Adverse effect of a treatment & $1.333(0.508-3.495)$ & 0.559 \\
\hline \multicolumn{3}{|l|}{ Extraintestinal manifestations* } \\
\hline Peripheral arthritis/arthralgia & $0.687(0.329-1.437)$ & 0.319 \\
\hline Uveitis/iritis & $2.226(0.839-5.904)$ & 0.108 \\
\hline Pyoderma gangrenosum & $3.031(0.374-24.568)$ & 0.299 \\
\hline Erythema nodosum & $1.661(0.493-5.592)$ & 0.413 \\
\hline Aphthous oral ulcers/stomatitis & $0.296(0.040-2.186)$ & 0.233 \\
\hline Ankylosing spondylitis & Omitted since it predicts 'no CC' perfectly & - \\
\hline Primary sclerosing cholangitis & $5.478(0.624-48.103)$ & 0.125 \\
\hline Other & Omitted since it predicts 'no CC' perfectly & \\
\hline
\end{tabular}

* For each complication or EIM, the value 'no' is the reference.

\section{Factors Associated with CC-Genotype}

In a first step, we assessed whether disease characteristics differ between homozygous carriers of the PTPN2 variant allele (CC genotype), when compared to heterozygous carriers (CT genotype) and non-carriers (TT genotype). In CD patients, univariate logistic regression analysis revealed that the CC genotype was associated with a higher age at first diagnosis (OR 1.315; 95\% CI 1.068$1.620 ; \mathrm{p}=0.010)$ and the existence of gallstones (OR 4.793; 95\% CI 2.000-11.490; $\mathrm{p}<0.001$; table 2).
In the subsequent multiple regression analysis, these 2 factors of the CC-genotype remained statistically significant. Moreover, the disease location, in particular colonic disease (L2) localization, was found to be close to reach significance in the univariate regression model $(p=0.069)$. Colonic disease localisation was significantly correlated with the CC genotype when considering the age at diagnosis and the presence of gallstones in the analysis (OR 2.620; 95\% CI 1.054-6.514; $\mathrm{p}<0.038$; table 3). 
Table 3. Multiple regression analysis to identify factors associated with the CC genotype of CD patients, UC patients, or all patients together as indicated. Although all parameters listed in online supplementary table 2 have been tested, only factors contributing to significance are depicted here

\begin{tabular}{llll}
\hline & OR & $95 \%$ CI & p value \\
\hline $\begin{array}{l}\text { CD patients } \\
\text { Age at diagnosis (decades) }\end{array}$ & 1.306 & $1.055,1.616$ & 0.014 \\
$\begin{array}{l}\text { Presence of gallstones } \\
\text { Current CD location }\end{array}$ & 4.707 & $1.923,11.523$ & 0.001 \\
$\quad$ L1 & 1 (ref.) & & \\
$\quad$ L2 & 2.620 & $1.054,6.514$ & 0.038 \\
$\quad$ L3 & 1.733 & $0.670,4.483$ & 0.257 \\
\hline $\begin{array}{l}\text { UC patients* } \\
\text { Current disease location is pancolitis }\end{array}$ & 4.938 & $1.366,17.857$ & 0.015 \\
\hline $\begin{array}{l}\text { All patients } \\
\text { Age at diagnosis, years }\end{array}$ & & \\
Presence of gallstones & 1.021 & $1.003,1.039$ & 0.024 \\
\hline \multicolumn{2}{l}{$*$ IC patients are excluded in this analysis. } & & 0.002 \\
\hline
\end{tabular}

\begin{tabular}{lllrl}
\hline Distribution of pancolitis* & TT & CT & \multicolumn{1}{l}{ CC } & \multicolumn{1}{l}{ Total } \\
\hline Pancolitis** & $209(67.9)$ & $88(28.6)$ & $11(3.6)$ & $308(100)$ \\
Other location** & $293(72.7)$ & $107(26.6)$ & $3(0.7)$ & $403(100)$ \\
\hline
\end{tabular}

* Only UC patients with known disease location have been included in the analysis.

** According to a Fisher's exact test, these 2 distributions are significantly different $(\mathrm{p}=0.020)$.
Table 4. Distribution of genotypes in UC patients with pancolitis compared to other disease locations
When analysing all UC/IC patients, pancolitis was the only factor that was significantly associated with the CCgenotype (OR 4.581; 95\% CI 1.267-16.556; $\mathrm{p}=0.020$ ). Of note, the results were similar when IC patients were not included in the analysis (OR 4.938; 95\% CI 1.366-17.857; $\mathrm{p}=0.015$; table 3 ). Further, the distribution of genotypes was different when comparing patients suffering from pancolitis with patients displaying another disease location $(\mathrm{p}=0.020$; table 4$)$.

\section{Factors Associated with the Presence of the C-Allele}

For some genes, the presence of one variant allele has an effect on protein function, and heterozygous carriers are similarly affected as homozygous carriers (dominant inheritance) $[18,19]$. Hence, in a next step, we assessed whether the presence of the $\mathrm{C}$-allele (including genotypes CC and CT) in PTPN2 affects clinical parameters. Grouping all CD patients with a $\mathrm{C}$-allele together revealed that uveitis was positively associated with the C-allele (OR 2.020; 95\% CI 1.201-3.398; $\mathrm{p}=0.008$ ), while aphthous oral ulcers revealed a negative association (OR 0.578; 95\% CI 0.335-0.998; $\mathrm{p}=$ $0.049)$. Although the $p$ value was at the borderline to statis- tical significance for both correlations, this remained significant in a multiple regression model, while no additional parameters were found to add significance when included in the multiple regression analysis (table 5).

When all UC/IC patients carrying the C-allele (CC and CT genotype) were compared to non-carriers (TT variant), we found that the age at diagnosis was higher in patients carrying the C-allele (OR 1.122; 95\% CI 1.001-1.257; $\mathrm{p}=0.049$; fig. 1$)$, while disease-related intestinal surgery (OR 3.056; 95\% CI 1.011-9.239; $\mathrm{p}=0.048$ ) was more common in $\mathrm{C}$-allele carriers. Again, the $\mathrm{p}$ value was marginally below the significance threshold of $p=0.05$, but here also the combination of the individually significant factors in a multiple logistic regression confirmed significance. Further, when IC patients were excluded from the analysis, the difference was even more significant ( $p=0.02$; table 5), additionally confirming our initial finding.

\section{Genotype Distribution Regarding Treatment Characteristics}

The distribution of genotypes (CC, CT and TT) was separately analysed for various treatment characteristics 
Table 5. Multiple regression analysis of $\mathrm{CD}, \mathrm{UC} / \mathrm{IC}$ and $\mathrm{UC}$ patients for factors associated with presence of the $\mathrm{C}$-allele

\begin{tabular}{lccc}
\hline & OR & $95 \%$ CI & p value \\
\hline CD & & & \\
$\begin{array}{l}\text { EIM } \\
\quad \text { Uveitis }\end{array}$ & 2.020 & $1.201-3.398$ & 0.008 \\
$\quad$ Aphthous oral ulcers & 0.578 & $0.335-0.998$ & 0.049 \\
\hline $\begin{array}{l}\text { UC/IC } \\
\text { Age at diagnosis (decades) }\end{array}$ & 1.122 & $1.001-1.257$ & 0.049 \\
Intestinal surgery & 3.056 & $1.011-9.239$ & 0.048 \\
\hline $\begin{array}{l}\text { UC (excluding IC) } \\
\text { Age at diagnosis, years }\end{array}$ & 1.014 & $1.002-1.026$ & 0.020 \\
$\begin{array}{l}\text { EIM - peripheral arthritis } \\
\text { Current disease location is }\end{array}$ & 1.394 & $0.961-2.022$ & 0.080 \\
$\quad$ pancolitis & 1.382 & $1.001-1.928$ & 0.049 \\
\hline
\end{tabular}

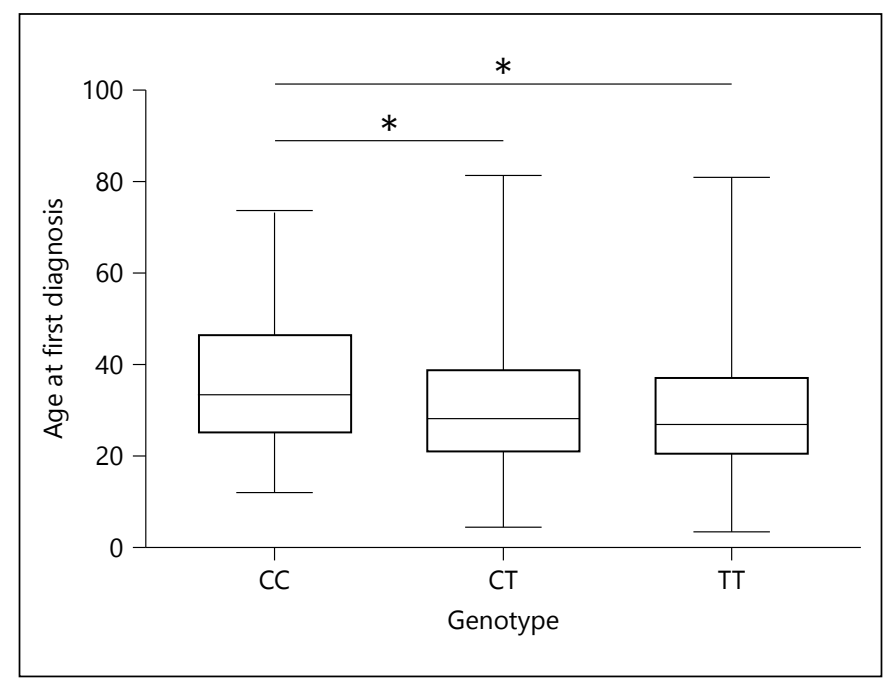

Fig. 1. The CC-genotype results in increased age of first diagnosis. The graph depicts distribution of age at first diagnosis, stratified according to PTPN2 genotype in SNP rs1893217. The boxes depict the following information: median (middle line), 25th-75th quartile (box borders), minimum-maximum (whiskers).

such as medication, response to this medication and IBDrelated intestinal surgery. In some cases, this distribution was significantly different from the global distribution of genotypes among our patient collective.

All patients who ever received anti-TNF antibody therapy were analysed according to their treatment response. Distribution of genotypes for those patients with response to anti-TNF therapy (7 CC/37 CT/71 TT) significantly differed from the overall distribution (53
CC/493 CT/1,297 TT; $\mathrm{p}$ value 0.05 , chi-square test; table 6; fig. 2). A similar result was obtained, considering the presence of $\mathrm{C}$-allele (CT and CC genotype combined; $\mathrm{p}$ value 0.05 ; table 6 ). When analysing patients stratified according to the specific anti-TNF antibody, similar results were obtained for patients receiving infliximab, that is, CC and CT carriers tended to respond more often to infliximab treatment than patients homozygous for the $\mathrm{T}$ allele (table 6). Due to a smaller sample size, however, this effect did not reach statistical significance. Only 2 patients who received adalimumab responded to the treatment and none of the patients who received certolizumab pegol responded to treatment; we therefore cannot conclude whether the presence of the $\mathrm{C}$ allele affects the response to these 2 drugs. Of note, however, the adalimumab responders were both heterozygous carriers of the C allele (table 6).

For other parameters such as the response to treatment with steroids or the use of immunomodulators, no significant difference was found regarding the distribution of genotypes.

\section{Discussion}

In this study, we used prospectively obtained patient data from the SIBDCS to analyse the clinical impact of SNP rs1893217 in the PTPN2 gene with respect to disease course and treatment response. It has been suggested that SNP rs1893217 influences the mRNA expression and enzymatic activity of PTPN2 [4]. The consequences of an altered mRNA and protein expression as well as enzymatic activity of PTPN2 have been well described in many in vitro and in vivo experiments and a possible role for PTPN2 in chronic inflammatory disorders has been suggested $[4,8,11-15]$. Given the growing evidence that PTPN2 is crucially involved in the pathogenesis of IBD, it is of great interest to determine whether the presence of genetic polymorphisms resulting in altered PTPN2 function, might affect the clinical course of IBD. Our study adds important new findings to the existing body of knowledge about PTPN2. With the consideration and analysis of disease and treatment characteristics, we successfully connected the biomedical understanding of a PTPN2 polymorphism with the clinical appearance of IBD. A further strength of this study is the large and standardized data set that was available from the prospective multi-centre nationwide SIBDC. This data represent a typical Western population and therefore enables direct comparison with many other studies in the field. 
Table 6. Association of PTPN2 SNP rs1893217 with anti-TNF therapy

\begin{tabular}{|c|c|c|c|c|}
\hline Genotype - all patients, $\%$ & $\mathrm{CC}$ & CT & TT & Total \\
\hline \multicolumn{5}{|l|}{ Therapy with anti-TNF } \\
\hline No & $40(2.6)$ & $417(26.5)$ & $1,114(70.9)$ & 1,571 \\
\hline Yes & $13(4.8)$ & $76(27.9)$ & $83(67.3)$ & 272 \\
\hline Total & $53(2.9)$ & $493(26.8)$ & $1,297(70.4)$ & 1,843 \\
\hline \multicolumn{5}{|l|}{ Anti-TNF therapy failed } \\
\hline No & $7(6.1)$ & $37(32.2)$ & $71(61.7)$ & 115 \\
\hline Yes & $5(3.3)$ & $39(25.5)$ & $109(71.2)$ & 153 \\
\hline Total & $12(4.5)$ & $76(28.4)$ & $180(67.2)$ & 268 \\
\hline Existence of C-allele - all patients, $\%$ & & Yes & No & Total \\
\hline \multicolumn{5}{|l|}{ Therapy with anti-TNF } \\
\hline No & & $457(29.1)$ & $1,114(70.9)$ & 1,571 \\
\hline Yes & & $89(32.7)$ & $183(67.3)$ & 272 \\
\hline Total & & $546(29.6)$ & $1,297(70.4)$ & 1,843 \\
\hline \multicolumn{5}{|l|}{ Anti-TNF therapy failed } \\
\hline No & & $44(38.3)$ & $71(61.7)$ & 115 \\
\hline Yes & & $44(28.8)$ & $109(71.2)$ & 153 \\
\hline Total & & $88(32.8)$ & $180(67.2)$ & 268 \\
\hline Genotype - CD patients, $\%$ & $\mathrm{CC}$ & CT & $\mathrm{TT}$ & Total \\
\hline \multicolumn{5}{|l|}{ Therapy with anti-TNF } \\
\hline No & $26(3.0)$ & $226(25.9)$ & $621(71.1)$ & 873 \\
\hline Yes & $12(6.0)$ & $56(28.0)$ & $132(66.0)$ & 200 \\
\hline Total & $38(3.5)$ & $282(26.3)$ & $753(70.2)$ & 1,073 \\
\hline \multicolumn{5}{|l|}{ Anti-TNF therapy failed } \\
\hline No & $6(6.6)$ & $28(30.8)$ & $57(62.6)$ & 91 \\
\hline Yes & $5(4.7)$ & $28(26.4)$ & $73(68.9)$ & 106 \\
\hline Total & $11(5.6)$ & $56(28.4)$ & $130(66.0)$ & 197 \\
\hline Existence of C-allele - CD patients, $\%$ & & Yes & No & Total \\
\hline \multicolumn{5}{|l|}{ Therapy with anti-TNF } \\
\hline No & & $252(28.9)$ & $621(71.1)$ & 873 \\
\hline Yes & & $68(34.0)$ & $132(66.0)$ & 200 \\
\hline Total & & $320(29.8)$ & $753(70.2)$ & 1,073 \\
\hline \multicolumn{5}{|l|}{ Anti-TNF therapy failed } \\
\hline No & & $34(37.4)$ & $57(62.6)$ & 91 \\
\hline Yes & & $33(31.1)$ & $73(68.9)$ & 106 \\
\hline Total & & $67(34.0)$ & $130(66.0)$ & 197 \\
\hline Genotype - UC patients*, \% & $\mathrm{CC}$ & $\mathrm{CT}$ & TT & Total \\
\hline \multicolumn{5}{|l|}{ Therapy with anti-TNF } \\
\hline No & $14(2.1)$ & $182(27.4)$ & $469(70.5)$ & 665 \\
\hline Yes & $1(1.5)$ & $19(27.5)$ & $49(71.0)$ & 69 \\
\hline Total & $15(2.0)$ & $211(27.4)$ & $544(70.7)$ & 734 \\
\hline \multicolumn{5}{|l|}{ Anti-TNF therapy failed } \\
\hline No & $1(4.8)$ & $8(38.1)$ & $12(57.1)$ & 21 \\
\hline Yes & $0(0.0)$ & $11(23.4)$ & $36(76.6)$ & 47 \\
\hline Unknown & & & 1 & 1 \\
\hline Total & $1(1.5)$ & $20(27.5)$ & $49(71.0)$ & 69 \\
\hline
\end{tabular}


Table 6. (continued)

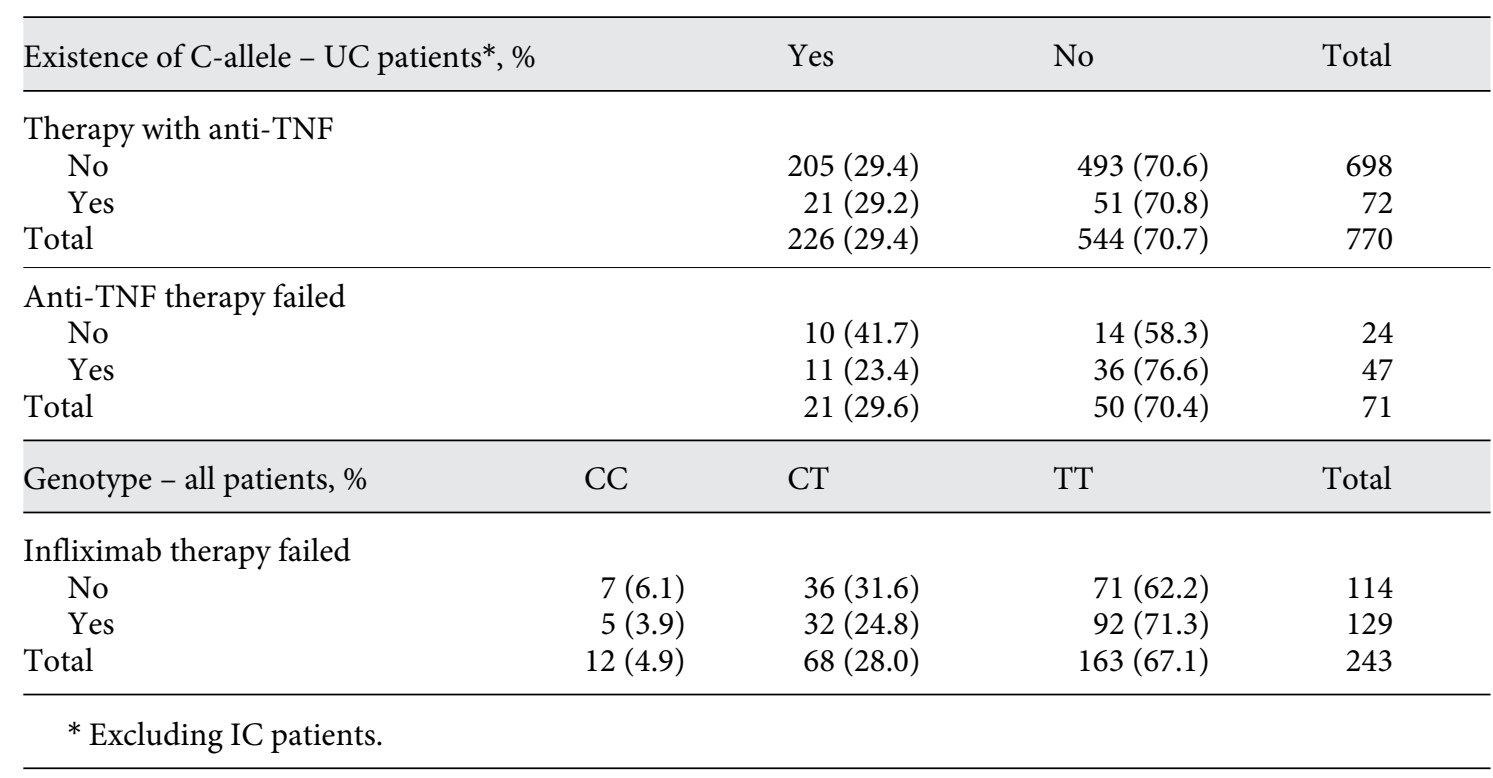

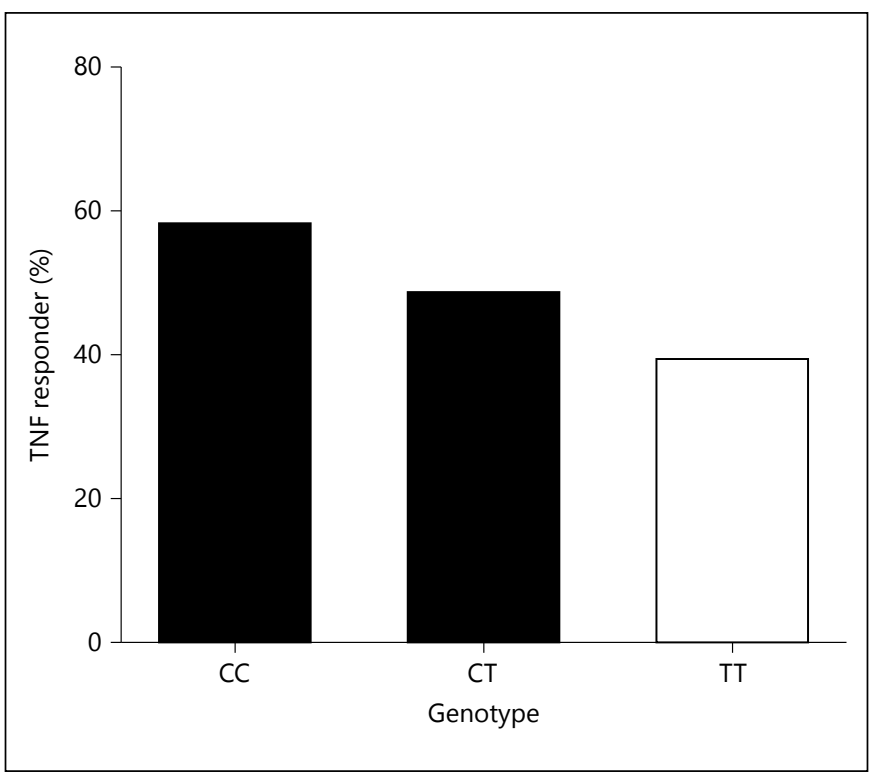

Fig. 2. Patients carrying the $\mathrm{C}$-allele respond more often to antiTNF treatment. The graph represents a percentage of patients carrying the indicated genotype in SNP rs1893217, who responded to anti-TNF treatment.

We found that both, $\mathrm{CD}$ and UC/IC patients carrying the CC-genotype were older at the onset of disease. Despite this later disease onset, we found that UC/IC patients carrying the CC-genotype suffered from a more extensive disease, namely, pancolitis. As UC characteristi- cally develops from the distal to the proximal colon, pancolitis indicates an aggravated and extensive disease. These findings are further supported by the fact that UC/ IC patients who carried a C-allele - despite later disease onset - were more likely to require intestinal surgery, mostly proctocolectomy. Pancolitis and EIM such as peripheral arthritis have been associated with active and advanced disease and might add up to an aggravated disease course in the presence of the C-allele [20-22]. Additionally, peripheral arthritis has been linked to pancolitis [23, 24]. The finding that aphthous oral ulcers was less frequent in C-allele carriers does not argue against our hypothesis, as current literature does not suggest an association between aphthous oral ulcers and disease activity $[20,25]$. Consequently, our results suggest that UC/IC patients homozygous for the $\mathrm{C}$-allele are prone to a more rapid, severe disease course.

In $\mathrm{CD}$, we found the existence of a C-allele to be a risk factor for uveitis but also protective for aphthous oral ulcers. In analogy to the C-allele in UC/IC, currently we cannot fully explain these findings as both EIM have been reported to actually often occur together [20]. This discrepancy could at least partially be explained by the fact that aphthous oral ulcers are influenced by a variety of other factors unrelated to IBD [26].

$\mathrm{CD}$ patients carrying the CC genotype suffered more often from gallstones. Formation of gallstones is a typical complication in $\mathrm{CD}$, partially due to an impaired enterohepatic circulation of bile salts [27] and a downregulated 
ileal bile acid uptake transporter [28]. Severe disease with widespread inflammation in the ileum limits the surface for reabsorption of bile salts to a minimum and could therefore be explanatory for an increased risk for gallstones. All in all, the presence of the C-allele is associated with a more severe disease course. This manifests in extensive intestinal disease, the need for intestinal surgery, a higher prevalence of certain EIM and characteristic disease-related complications.

In our study population, $\mathrm{CD}$ patients with this risk variant were more likely to respond to anti-TNF antibody treatment. Results from previous studies proved PTPN2 to be a key regulator of the effects of the pro-inflammatory cytokine TNF, and a negative feedback loop between TNF and PTPN2 is presumed $[9,29]$. It is known that TNF increases the expression of PTPN2 in intestinal epithelial cells [9]. In reverse, PTPN2 is an important inhibitor of the secretion of proinflammatory cytokines like TNF. This has been confirmed by detecting significantly higher TNF levels in PTPN2-deficient mice [30, 31]. We have previously shown that the presence of the PTPN2 SNP rs1893217 causes a diminished function of PTPN2 protein [4]. In that way, the inhibitory signals for TNF in the negative feedback loop are weakened and consequently more TNF is released. TNF reaches higher serum levels in patients with autoimmune diseases and is an essential pro-inflammatory cytokine that maintains chronic inflammation [31-33]. By blocking the excessive TNF production with anti-TNF agents, this viscous inflammation-maintaining cycle can be interrupted. This could explain our observed higher response rate to anti-TNF in patients with a C-allele compared to those without.

Our results are of interest for pharmacotherapy of IBD, as they might prove the legitimation for early use of anti-TNF antibodies in certain patient subgroups, and might help identify patients that are more susceptible to anti-TNF treatment. To date, in daily practice, anti-TNF antibodies are mainly used in IBD patients not responding to or being intolerant to steroids and immunomodulators, also known as the 'step-up' approach $[34,35]$. Unfortunately, a decade of experience with anti-TNF antibodies indicates that about one third of IBD patients are anti-TNF antibody non-responders [36-40]. Although many factors such as young age at diagnosis, non-smoking (OR 2.33 [41]), pANCA negativity (OR 1.87 [42]), and various gene expression profiles (PTPN2 polymorphisms not included) have been associated with a higher response rate, so far none of them has been clearly implemented in clinical decision making [39, 41-44]. As the use of anti-TNF antibodies is constantly increasing, the elaboration of a model for prediction of response is of a major interest. Considering our results and the current available literature, a multifactorial model that includes PTPN2 polymorphisms might be the most promising predictive approach to achieve this. Nevertheless, our data might suggest the early use of anti-TNF antibodies in UC patients carrying the disease-associated PTPN2 variant. However, since patient numbers of $\mathrm{CD}$ patients with the CC genotype treated with anti-TNF antibodies were low, and our entire data were obtained only from a single cohort, validation of our findings in an independent patient cohort or in a prospective trial would clearly be necessary before drawing a final conclusion on the effect of the C-allele regarding anti-TNF medication.

After all, there are some methodological limitations in this study. UC patients with a particularly severe course of disease might be over-represented in our UC patient collective, since in general, more patients with a severe disease course are included in the SIBDCS and a significant number of patients with a mild disease course might not be represented in this cohort. Furthermore, we face a selection bias that applies to most cohort studies: difficult cases tend to converge in specialized IBD clinics, while mild cases are commonly treated at private practice gastroenterologists. Although our cohort features a fraction of IBD patients receiving care in gastroenterology private practice (about $30 \%$ of patients), those IBD patients treated in secondary or tertiary referral centres are somewhat overrepresented in our cohort. Additionally, the awareness of studies is naturally higher at the institution where they are conducted. It is thus likely that blood samples for genotyping were obtained more frequently at our clinic than in district hospitals or gastroenterology private practices. Further, our data were generated exclusively in the SIBDC. Although the SIBDC is very well characterized and represents a typical European cohort [16], validation of our finding in additional, independent cohorts will be necessary to confirm our findings. Nevertheless, since only few data exist that describe a clinical relevance of IBD candidate genes, our findings might critically contribute to a better understanding of the complex interaction between genetic predispositions and the clinical appearance of IBD.

In summary, this is the first study that showed that IBD patients homozygous or heterozygous for the PTPN2 SNP rs1893217 were at greater risk for developing a severe course of disease but were more likely to respond to treatment with anti-TNF antibodies. However, to draw a definite conclusion regarding treatment recommendations for patients with PTPN2 polymorphisms, our results need to be confirmed by further clinical studies. 


\section{Acknowledgements}

The authors thank all the patients for their collaboration and the members of the SIBDCS for their contribution.

\section{Financial Support}

This research was supported by research grants from the Swiss National Science Foundation to M.R.S. (grant Nos. 314730-146204 and CRSII3_154488/1) and to G.R. for the Swiss IBD Cohort (grant No. 3347CO-108792). The funding institutions had no role in study design and data interpretation.

\section{Potential Competing Interests}

None.

\section{Author Contributions}

M.R.S. and M.V. wrote the manuscript and interpreted the data, J.-B.R. performed statistical analysis, S.R.V. and M.C.S. conceived the study, M.C.S. designed and supervised the study and corrected the manuscript. All other authors were involved in data acquisition and data interpretation. All authors approved the final submitted draft.

\section{Members of the SIBDCS Group}

Claudia Anderegg; Peter Bauerfeind; Christoph Beglinger; Stefan Begré; Dominique Belli; José Bengoa; L. Biedermann; Janek Binek; Mirjam Blattmann; Nadia Blickenstorfer; Stephan Boehm; Jan Borovicka; Christian Braegger; Patrick Bühr; Ber- nard Burnand; Emmanuel Burri; Sophie Buyse; Matthias Cremer; Dominique Criblez; Philippe de Saussure; Lukas Degen; Joakim Delarive; Christopher Dörig; Barbara Dora; Gian Dorta; Tobias Ehmann; Ali El Wafa; Mara Egger; Matthias Engelmann; Christian Felley; Markus Fliegner; Nicolas Fournier; Montserrat Fraga; Alain Frei; P. Frei; Remus Frei; M. Fried; Florian Froehlich; Raoul Furlano; Suzanne Gallot-Lavallée; Martin Geyer; Marc Girardin; Delphine Golay; Tanja Grandinetti; Beat Gysi; Horst Haack; Johannes Haarer; Beat Helbling; Peter Hengstler; Denise Herzog; Cyrill Hess; Klaas Heyland; Thomas Hinterleitner; Philippe Hiroz; Claudia Hirschi; Petr Hruz; Pascal Juillerat; Rosmarie Junker; Christina Knellwolf; Christoph Knoblauch; Henrik Köhler; Rebekka Koller; Claudia Krieger; Gerd A. Kullak-Ublick; Markus Landolt; Frank Lehmann; Valérie McLin; Philippe Maerten; Michel Maillard; Christine Manser; Andrew Macpherson; Michael Manz; George Marx; Rémy Meier; Christa Meyenberger; Jonathan Meyer; Pierre Michetti; Benjamin Misselwitz; Darius Moradpour; Patrick Mosler; Christian Mottet; Christoph Müller; Pascal Müller; Beat Müllhaupt; Claudia Münger; Leilla Musso; Andreas Nagy; Cristina Nichita; Jan Niess; Natacha Noël; Andreas Nydegger; Maliza Nzabonimpa; Nicole Obialo; Carl Oneta; Cassandra Oropesa; Céline Parzanese; Laetitia-Marie Petit; Franziska Piccoli; Julia Pilz; Gaëlle Pittet; Valérie Pittet; Bruno Raffa; Ronald Rentsch; Sophie Restellini; Jean-Pierre Richterich; Silvia Rihs; Jocelyn Roduit; Daniela Rogler; G. Rogler; J.-B. Rossel; Markus Sagmeister; Gaby Saner; Bernhard Sauter; Mikael Sawatzki; M. Scharl; S. Scharl; Nora Schaub; Martin Schelling; Susanne Schibli; Hugo Schlauri; Daniela Schmid; Sybille Schmid; Jean-François Schnegg; Alain Schoepfer; Christiane Sokollik; Frank Seibold; Gian-Marco Semadeni; Mariam Seirafi; David Semela; Arne Senning; Marc Sidler; Johannes Spalinger; Holger Spangenberger; Philippe Stadler; Volker Stenz; Michael Steuerwald; Alex Straumann; M.C. Sulz; Alexandra Suter; Michela Tempia-Caliera; Joël Thorens; Sarah Tiedemann; Radu Tutuian; Ueli Peter; S.R. Vavricka; Francesco Viani; Roland Von Känel; Alain Vonlaufen; Dominique Vouillamoz; Rachel Vulliamy; Helene Werner; Paul Wiesel; Reiner Wiest; Tina Wylie; J. Zeitz; Dorothee Zimmermann.

\section{References}

1 Jostins L, et al: Host-microbe interactions have shaped the genetic architecture of inflammatory bowel disease. Nature 2012;491:119-124.

2 Liu JZ, et al: Association analyses identify 38 susceptibility loci for inflammatory bowel disease and highlight shared genetic risk across populations. Nat Genet 2015;47:979-986.

3 Genome-wide association study of 14,000 cases of seven common diseases and 3,000 shared controls. Nature 2007;447:661-678.

4 Scharl M, et al: Crohn's disease-associated polymorphism within the PTPN2 gene affects muramyl-dipeptide-induced cytokine secretion and autophagy. Inflamm Bowel Dis 2012; 18:900-912.

5 Franke A, et al: Replication of signals from recent studies of Crohn's disease identifies previously unknown disease loci for ulcerative colitis. Nat Genet 2008;40:713-715.
6 Todd JA, et al: Robust associations of four new chromosome regions from genome-wide analyses of type 1 diabetes. Nat Genet 2007; 39:857-864.

7 Scharl M, et al: Protein tyrosine phosphatase nonreceptor type 2 regulates autophagosome formation in human intestinal cells. Inflamm Bowel Dis 2012;18:1287-1302.

8 Scharl M, et al: Protection of epithelial barrier function by the Crohn's disease associated gene protein tyrosine phosphatase N2. Gastroenterology 2009;137:2030-2040. e5.

9 Scharl M, et al: Protein tyrosine phosphatase $\mathrm{N} 2$ regulates $\mathrm{TNF} \alpha$-induced signalling and cytokine secretion in human intestinal epithelial cells. Gut 2011;60:189-197.

10 Scharl M, Hruz P, McCole DF: Protein tyrosine phosphatase non-receptor type 2 regu- lates IFN- $\gamma$-induced cytokine signaling in THP-1 monocytes. Inflamm Bowel Dis 2010; 16:2055-2064.

11 Heinonen KM, et al: T-cell protein tyrosine phosphatase deletion results in progressive systemic inflammatory disease. Blood 2004; 103:3457-3464.

12 You-Ten KE, et al: Impaired bone marrow microenvironment and immune function in $\mathrm{T}$ cell protein tyrosine phosphatasedeficient mice. J Exp Med 1997;186:683693.

13 Wiede $\mathrm{F}$, et al: $\mathrm{T}$ cell protein tyrosine phosphatase attenuates $\mathrm{T}$ cell signaling to maintain tolerance in mice. J Clin Invest 2011;121: 4758-4774.

14 Wiede F, La Gruta NL, Tiganis T: PTPN2 attenuates T-cell lymphopenia-induced proliferation. Nat Commun 2014;5:3073. 
15 Spalinger MR, et al: PTPN2 controls differentiation of $\mathrm{CD}^{+} \mathrm{T}$ cells and limits intestinal inflammation and intestinal dysbiosis. Mucosal Immunol 2015;8:918-929.

16 Pittet V, et al: Cohort profile: the Swiss inflammatory bowel disease cohort study (SIBDCS). Int J Epidemiol 2009;38:922-931.

17 Silverberg MS, et al: Toward an integrated clinical, molecular and serological classification of inflammatory bowel disease: report of a working party of the 2005 Montreal world congress of gastroenterology. Can J Gastroenterol 2005;19(suppl A):5A-36A.

18 Veitia RA, Bottani S, Birchler JA: Gene dosage effects: nonlinearities, genetic interactions, and dosage compensation. Trends Genet 2013;29:385-393.

19 Chou CM, et al: Biochemical basis for dominant inheritance, variable penetrance, and maternal effects in RBP4 congenital eye disease. Cell 2015;161:634-646.

20 Vavricka SR, et al: Frequency and risk factors for extraintestinal manifestations in the Swiss inflammatory bowel disease cohort. Am J Gastroenterol 2011;106:110-119.

21 Barrie A, Regueiro M: Biologic therapy in the management of extraintestinal manifestations of inflammatory bowel disease. Inflamm Bowel Dis 2007;13:1424-1429.

22 Greenstein AJ, Janowitz HD, Sachar DB: The extra-intestinal complications of Crohn's disease and ulcerative colitis: a study of $700 \mathrm{pa}-$ tients. Medicine (Baltimore) 1976;55:401412.

23 Dorofeyev AE, Vasilenko IV, Rassokhina OA: Joint extraintestinal manifestations in ulcerative colitis. Dig Dis 2009;27:502-510.

24 Monsén U, et al: Extracolonic diagnoses in ulcerative colitis: an epidemiological study. Am J Gastroenterol 1990;85:711-716.
25 Lankarani KB, Sivandzadeh GR, Hassanpour $S$ : Oral manifestation in inflammatory bowel disease: a review. World J Gastroenterol 2013; 19:8571-8579.

26 McCullough MJ, Abdel-Hafeth S, Scully C: Recurrent aphthous stomatitis revisited; clinical features, associations, and new association with infant feeding practices? J Oral Pathol Med 2007;36:615-620.

27 Hutchinson R, et al: Pathogenesis of gall stones in Crohn's disease: an alternative explanation. Gut 1994;35:94-97.

28 Jahnel J, et al: Inflammatory bowel disease alters intestinal bile acid transporter expression. Drug Metab Dispos 2014;42:1423-1431.

29 van Vliet C, et al: Selective regulation of tumor necrosis factor-induced Erk signaling by Src family kinases and the T cell protein tyrosine phosphatase. Nat Immunol 2005;6:253260.

30 Hassan SW, et al: Increased susceptibility to dextran sulfate sodium induced colitis in the $\mathrm{T}$ cell protein tyrosine phosphatase heterozygous mouse. PLoS One 2010;5:e8868.

31 Solus JF, et al: Genetics of serum concentration of IL- 6 and TNFa in systemic lupus erythematosus and rheumatoid arthritis: a candidate gene analysis. Clin Rheumatol 2015;34: 1375-1382.

32 MacDonald TT, et al: Tumour necrosis factor-alpha and interferon-gamma production measured at the single cell level in normal and inflamed human intestine. Clin Exp Immunol 1990;81:301-305.

33 Reinecker HC, et al: Enhanced secretion of tumour necrosis factor-alpha, IL-6, and IL-1 beta by isolated lamina propria mononuclear cells from patients with ulcerative colitis and Crohn's disease. Clin Exp Immunol 1993;94: 174-181.

34 Van Assche G, et al: The second European evidence-based consensus on the diagnosis and management of Crohn's disease: special situations. J Crohns Colitis 2010;4:63-101.
35 Dignass A, et al: Second European evidencebased consensus on the diagnosis and management of ulcerative colitis part 2: current management. J Crohns Colitis 2012;6:9911030.

36 Rutgeerts $\mathrm{P}$, et al: Infliximab for induction and maintenance therapy for ulcerative colitis. N Engl J Med 2005;353:2462-2476.

37 Cohen RD, Tsang JF, Hanauer SB: Infliximab in Crohn's disease: first anniversary clinical experience. Am J Gastroenterol 2000;95: 3469-3477.

38 Ljung $\mathrm{T}$, et al: Infliximab in inflammatory bowel disease: clinical outcome in a population based cohort from Stockholm County. Gut 2004;53:849-853.

39 Oussalah A, et al: A multicenter experience with infliximab for ulcerative colitis: outcomes and predictors of response, optimization, colectomy, and hospitalization. Am J Gastroenterol 2010;105:2617-2625.

40 Gavalas E, et al: Efficacy and safety of infliximab in steroid-dependent ulcerative colitis patients. Hepatogastroenterology 2007;54: 1074-1079.

41 Bank S, et al: Effectiveness of anti-tumour necrosis factor- $\alpha$ therapy in Danish patients with inflammatory bowel diseases. Dan Med J 2015;62:pii:A4994..

42 Nguyen DL, Nguyen ET, Bechtold ML: pANCA positivity predicts lower clinical response to infliximab therapy among patients with IBD. South Med J 2015;108:139-143.

43 Arijs I, et al: Mucosal gene signatures to predict response to infliximab in patients with ulcerative colitis. Gut 2009;58:1612-1619.

44 Arijs I, et al: Predictive value of epithelial gene expression profiles for response to infliximab in Crohn's disease. Inflamm Bowel Dis 2010; 16:2090-2098. 